\title{
Expansión educativa e inmigración internacional en España: cambios en la formación de la pareja ${ }^{1}$
}

\author{
Albert Esteve* \\ Clara Cortina** \\ *Universitat Autònoma de Barcelona. Centre d'Estudis Demogràfics \\ aesteve@ced.uab.es \\ ${ }^{* *}$ Consejo Superior de Investigaciones Científicas \\ clara.cortina@cchs.csic.es
}

Recibido: 10-10-2007

Aceptado: 05-05-2008

\section{Resumen}

En este artículo, estudiamos la composición de las parejas españolas, según el nivel de instrucción y el origen de los cónyuges, en un contexto caracterizado por la expansión educativa de la población española y el incremento de la población extranjera. El estudio de las generaciones nacidas entre 1920 y 1969 realizado a partir del censo de población de 2001 desvela una reducción de los niveles generales de homogamia educativa de generación en generación, una intensificación de la homogamia de los cónyuges con estudios superiores, una pérdida reciente de vigencia de la pauta de hipergamia educativa y una importante variabilidad de dichas pautas entre los extranjeros.

Palabras clave: homogamia educativa, población extranjera, nivel de instrucción, nacionalidad, matrimonio, España, censo de 2001.

Abstract. Educational expansion and international migration in Spain: changes in the patterns of the formation of partnerships

This article analyses patterns and trends in educational assortative mating and intermarriage of Spanish couples born between 1920 and 1969 using data from the 2001 Spanish Census. We examine those changes in a context of educational expansion and dramatic increase of foreign population in Spain since the mid-nineties. The most notable trends are the decrease of global homogamy levels across cohorts, which contrasts with the increasing intensity of homogamy among the more educated; the incipient extinction of the traditional female hypergamic pattern; and the differences observed among the different groups of foreigners regarding their assortative mating patterns.

Key words: assortative mating, foreign population, educational attainment, marriage, citi zenship, Spain, 2001 census.

1. Este trabajo se inscribe en el proyecto I+D+I del Ministerio de Educación y Ciencia El impacto de la población extranjera en la formación de la pareja en España (SEJ2007-60014). Una versión previa de este trabajo fue presentada en el Congreso Español de Sociología, celebrado en Barcelona en el mes de septiembre de 2007. Los autores quieren expresar aquí su agradecimiento a Pau Miret por sus valiosos comentarios. Albert Esteve es investigador Ramón y Cajal en el Centre d'Estudis Demogràfics. Clara Cortina es investigadora del Grupo de Investigación de Dinámicas Demográficas (GIDD) y del Grupo de Estudios de Población y Sociedad (GEPS). 


\author{
Sumario \\ Introducción Modelos loglinales \\ Consideraciones teóricas, evidencia \\ empírica e hipótesis de trabajo \\ Datos \\ Resultados \\ Conclusiones \\ Referencias bibliográficas
}

\section{Introducción}

A lo largo del siglo Xx, la sociedad española ha experimentado una espectacular transformación en su estructura académica. La universalización de la educación y el aumento de los niveles de formación constituyen unos de los elementos determinantes de la modernización social y económica del país. Muestra de ello es la reducción de la proporción de personas sin estudios primarios, que roza el 50\% en la generación nacida en la década de 1920 y se reduce a menos de un 5\% en la nacida en la década de 1960 . También destaca el significativo aumento de la proporción de personas con estudios universitarios, que alcanza aproximadamente el $20 \%$ para esta última generación. Junto a la expansión educativa, en la última década hemos asistido a un importante incremento de los flujos inmigratorios internacionales. Según datos del padrón municipal de habitantes, la población de nacionalidad extranjera ha crecido de 542.314 personas en 1996 a 3.730 .610 a inicios de 2005 , o lo que es lo mismo, en cifras relativas, del $1,4 \%$ al $8,5 \%$ del total de la población española. Además de la intensidad de este crecimiento, la estructura de edad particularmente joven de la población extranjera refuerza su impacto en la formación de la pareja.

Ante dichos cambios, el presente artículo analiza la composición de las parejas españolas de cónyuges nacidos entre 1920 y 1969, según el nivel de instrucción y el origen de sus miembros. En primer lugar, repasamos las referencias teóricas para el estudio de la homogamia de las parejas; en segundo lugar, presentamos los datos del censo de 2001, con el que realizaremos nuestro análisis, y la metodología utilizada, y, en tercer lugar, presentamos los principales resultados obtenidos en relación con los aspectos siguientes:

1) La continuidad y los cambios de los niveles de homogamia educativa a lo largo del siglo XX.

2) La vigencia de la pauta de hipergamia educativa femenina (modelo tradicional de matrimonio con un hombre más instruido que la mujer).

3) Las pautas de las parejas formadas por cónyuges extranjeros de cuatro grandes grupos (europeos comunitarios, europeos no comunitarios, africanos y latinoamericanos) para la generación de 1960-1969. 


\section{Consideraciones teóricas, evidencia empírica e hipótesis de trabajo}

\section{La formación de la pareja}

Si las personas nos emparejáramos al azar, con independencia de nuestro origen, religión, posición económica, estatus social u otra característica adscrita o adquirida, la probabilidad de formar una unión homógama estaría exclusivamente determinada por la distribución o la disponibilidad combinada de efectivos de las variables de referencia o, en otras palabras, por los condicionantes estructurales del mercado matrimonial. Sin embargo, entre los científicos sociales, existe un amplio consenso en torno a la consideración que, más allá del azar, individuos con características similares tienden a unirse entre ellos formando parejas homógamas (Kalmijn, 1998; McCaa, 1993; Mare, 1991; Blossfeld et al., 2003; Smits et al., 1998; Birkelund y Heldal, 2003; Carabaña, 1994). Si bien en el proceso de selección conyugal intervienen los determinantes estructurales, las preferencias personales y la mediación de terceras partes - históricamente: familia, estado e Iglesia- (Kalmijn, 1998), en las sociedades contemporáneas, donde formar pareja se convierte en un acto eminentemente voluntario, la mayoría de investigaciones hacen énfasis en las preferencias individuales.

Los individuos proceden a seleccionar una pareja de acuerdo con sus afinidades y sus preferencias individuales. Bajo un supuesto de racionalidad individual en la toma de decisiones afectivo-familiares, la «nueva economía de la familia», encabezada por el economista americano Gary Becker (1987), considera que estas preferencias buscan maximizar el intercambio de recursos que se produce en la formación de la pareja. Sin embargo, la satisfacción de estas preferencias mediante una opción óptima no siempre es posible. Ante un contexto de posibilidades matrimoniales limitadas y sometidas a la eventual presión de terceras partes, los candidatos se conforman con una buena opción. Nos referimos, de un lado, a la importancia que pueden tener los factores normativos familiares o sociales, que, si bien han perdido su hegemonía, pueden seguir ejerciendo su influencia mediante la definición de círculos de relación social que suponen un primer filtro, previo a la selección del cónyuge. Y nos referimos también, por otro lado, a la existencia de un mercado matrimonial que determina las opciones de los candidatos a casarse, esto es, de los futuros cónyuges. Los mercados matrimoniales son los espacios físicos y simbólicos de encuentro de aquellos hombres y mujeres en disposición de contraer matrimonio (Cabré, 1993) y ejercen como condicionantes de las opciones matrimoniales cuando se encuentran en situación de desequilibrio, es decir, cuando escasean los hombres o las mujeres, en su conjunto o con determinadas características.

Los investigadores se han centrado tanto en las implicaciones individuales como sociales de las pautas de composición de las parejas. Así, a nivel individual, la composición de las parejas se analiza en el marco del estudio de la familia, asumiendo que las características de los cónyuges y su combinación pueden determinar las decisiones familiares que éstos tomarán posteriormente (González, 2001); especialmente aquellas decisiones que conciernen tanto a 
la fecundidad como a las posibilidades de ruptura matrimonial. Por su parte, los sociólogos han hecho un énfasis especial en el papel del matrimonio en la reproducción de la estructura social (Kalmijn, 1991), entendiendo la formación de la pareja como un recurso utilizado por los individuos para consolidar o mejorar su condición social, tanto individual como familiar. En el marco de las implicaciones sociales, el matrimonio es considerado también como producto y agente de integración social. Desde este punto de vista, se ha estudiado ampliamente la interacción entre grupos sociales a partir de la formación de matrimonios entre miembros de distintos colectivos étnico-religiosos en contextos inmigratorios (McCaa, 1993; Pagnini y Morgan, 1990).

Dada la trascendencia del matrimonio, el estudio de la homogamia, en particular, ha captado el interés de varias disciplinas: la demografía, la antropología y, por supuesto, la sociología. Son homógamas las uniones que se caracterizan por integrar a dos cónyuges que comparten una característica determinada. En contraposición, las heterógamas unen a cónyuges dispares y pueden ser hipérgamas, cuando el cónyuge de referencia es menos que el consorte (es decir, que se une hacia arriba) o bien hipógamas, cuando el de referencia es más (y, por tanto, se une hacia abajo). Fundamentalmente, la semejanza de los cónyuges se analiza, en primer lugar, respecto a características adscritas, tales como la posición social definida por la adscripción familiar, la religión o la etnia, y, en segundo lugar, respecto a características adquiridas, entre las que destaca el nivel educativo. Las barreras matrimoniales existentes entre individuos de distintos grupos sociales, religiosos o étnicos pierden relevancia en las sociedades contemporáneas, mientras que los elementos de identificación sociales adquiridos, como el nivel de instrucción, son cada vez más determinantes (Kalmijn, 1998). Para entender este proceso, es importante entender la educación como una variable que no tan solo informa de la calificación de los individuos, sino también, indirectamente, de su posición socioeconómica y de su capacidad de promoción social y profesional. Por este motivo, el análisis de la concordancia entre los niveles educativos de los miembros de la pareja se ha convertido en una prioridad en el estudio de la homogamia.

\section{La homogamia educativa de las parejas}

Existen distintas teorías que consideran el papel que la educación puede tener como determinante de las opciones matrimoniales. Según la teoría clásica de la nueva economía de la familia, el modelo óptimo sería el de la pareja complementaria, aquélla en la que el hombre se especializa en tareas productivas y la mujer, en las reproductivas (Becker, 1987). De acuerdo con este esquema, el nivel educativo del marido sería más valorado que el de la esposa y, por consiguiente, se esperaría una tendencia a la hipergamia femenina. Sin embargo, como apunta Oppenheimer (1994), la incorporación de la mujer al mercado laboral obliga a replantear este principio de complementariedad, puesto que el trabajo extradoméstico adquiere valor específico. En este nuevo contexto, en el que las parejas optimizan sus recursos sin necesidad de especializarse, el 
nivel de instrucción de la mujer pasa a ser tan valorado como el del hombre, de tal manera que sería de esperar un aumento de los niveles de homogamia.

Otro elemento que apunta al crecimiento de la homogamia es, según Mare (1991), la ampliación del período de escolarización. Según esta hipótesis, para los individuos más educados, la permanencia prolongada en el sistema educativo maximiza las oportunidades de conocer y formar pareja con compañeros de estudio, es decir, con individuos de nivel educativo equivalente. Este efecto se refuerza por la coincidencia o proximidad de la edad de salida de la etapa educativa y la edad de formación de la pareja. Ambos elementos inciden en la tendencia a la homogamia matrimonial entre los más educados y, también, por consiguiente, en la de los demás grupos, que ven limitadas sus opciones de formar pareja con los niveles educativos superiores.

También han sido consideradas variables de tipo macroeconómico, con el propósito de vincular el nivel de desarrollo económico e industrial de los países con las pautas de composición de las parejas (Smits et al., 1998). En este marco de análisis, el nivel educativo adquiere valor en el proceso de selección de la pareja a medida que las sociedades se desarrollan económicamente y aumenta el individualismo. Este proceso acarrearía un incremento de los niveles de homogamia que tenderían a reducirse en una fase posterior, cuando la generalización de los niveles de bienestar restaría importancia al papel de la educación como garante del éxito socioeconómico.

Más allá de la teoría, la investigación llevada a cabo ha mostrado como los niveles generales de homogamia educativa han tendido a mantenerse constantes o bien a decrecer durante las últimas décadas del siglo XX en países europeos como Francia (Forsé y Chauvel, 1995), Gran Bretaña (Halpin y Chan, 2003), Noruega (Birkelund y Heldal, 2003), países de Europa central como la República Checa, Eslovaquia y Hungría (Katrnák, 2005) y también España (González, 2003; Cortina, 2004). Sin embargo, esta tendencia generalizada contrasta con la presentada por Schwartz y Mare (2005) para Estados Unidos, donde los niveles de homogamia habrían decrecido hasta 1960 para recuperarse a continuación, como consecuencia de un aumento de la homogamia de los grupos educativos extremos. Este aumento de la homogamia de los más instruidos (no así de los menos) se detecta en todos los países y se atribuye a los hechos siguientes:

1) $\mathrm{Al}$ incremento del número de hombres y mujeres con estudios superiores.

2) A la reducción del intervalo de tiempo entre el fin de la escolarización y la entrada en unión.

3) A la superación de las expectativas diferenciales por sexo.

En lo que se refiere a los análisis centrados en las diferencias entre hombres y mujeres en las pautas de hipergamia, los estudios más reveladores se han llevado a cabo en países donde persisten importantes diferencias educativas por sexo. En una comparación entre México y Brasil, Esteve y McCaa (2007) observan como el patrón clásico de hipergamia femenina se ha disuelto en Brasil, mientras que se mantiene vigente en México. En este sentido, Brasil se uniría a 
la trayectoria de otros países desarrollados, como España (Esteve y Cortina, 2006) o Estados Unidos (Qian, 1998), donde las diferencias de género en materia de hipergamia educativa se han ido suavizando hasta desaparecer.

\section{La endogamia de las parejas}

Los estudios de la formación de la pareja se han interesado también por la interacción matrimonial entre individuos de orígenes distintos y, por extensión, por la interacción social entre grupos. En la literatura anglosajona, este ámbito de estudio se conoce genéricamente bajo el epíteto general de intermarriage, noción que equivale a la pauta de exogamia, la de las uniones entre individuos pertenecientes a distintos grupos o colectivos. En este caso, la variable de referencia es una característica adscrita (el lugar de nacimiento) y no adquirida como en el caso del nivel de instrucción, aunque existen interacciones entre ellas. La hipótesis del intercambio social (social exchange) de Merton (1941) sugiere que los individuos que pertenecen a una minoría étnica utilizan su estatus socioeconómico como elemento de cambio para contrarrestar las desventajas sociales derivadas de su condición étnica o de raza. Esta hipótesis es de aplicación también en el caso de la formación de las parejas y se complementa con los planteamientos asimilacionistas clásicos (Gordon, 1964), tradicionalmente usados en los trabajos de intermarriage. Los asimilacionistas interpretan la formación de matrimonios exógamos como un elemento del proceso de acercamiento de los grupos minoritarios hacia el mayoritario, o de distintos grupos minoritarios entre sí (en el caso de la asimilación segmentada de Portes, 1993). Es decir, que además de las dinámicas de tipo grupal, la teoría del intercambio social introduce una dimensión individual, al considerar las características adscritas de cada individuo (y de su pareja respectiva) además del grupo de adscripción.

Los antecedentes empíricos se encuentran básicamente en la literatura sobre Estados Unidos y otros países de larga tradición inmigratoria y de marcada diversidad racial. Estos estudios ponen de relieve como, efectivamente, la educación tiene un papel variable en función de la combinación étnica de los cónyuges. Sin embargo, esta variabilidad se minimiza en las uniones de los más instruidos, que son los que mayor tendencia tienen a unirse entre sí. En este caso, la educación parecería actuar de atenuante de las diferencias por origen. Las pautas matrimoniales de los mexicanos en Estados Unidos son una buena ilustración de este fenómeno. En España, en cambio, por ser éste un país tradicionalmente homogéneo en lo que se refiere a su composición por origen, la literatura sobre intermarriage es escasa y sólo se ha empezado a desarrollar con la llegada de población extranjera (Cortina, Esteve y Domingo, 2006 y 2008).

\section{Hipótesis de trabajo}

En primer lugar, y como consecuencia de la diversificación de la estructura educativa, es de esperar una reducción del nivel general de homogamia edu- 
cativa a lo largo de las generaciones estudiadas. Sin embargo, apuntamos la posibilidad que los individuos con estudios superiores no experimenten dicha reducción, sino que, al contrario, su propensión a la homogamia aumente. Esta expectativa se basa en la hipótesis de una creciente simetría de género en los criterios de selección de la pareja (según Oppenheimer (1994), tanto hombres como mujeres buscan a los candidatos con mayor instrucción), así como en la mayor homogeneidad del mercado matrimonial de los universitarios (Mare, 1991).

En segundo lugar, teniendo en cuenta el valor adquirido por el nivel educativo de las mujeres y la equiparación de los niveles entre sexos, esperamos que la pauta tradicional de hipergamia femenina no se mantenga. Como ha ocurrido ya en otros países europeos, y especialmente en aquéllos en los que la expansión educativa ha reducido las diferencias de género, cada vez es más frecuente encontrar parejas en las que la mujer tiene más estudios que el hombre.

En tercer lugar, la heterogeneización de la población española en términos de origen o procedencia, derivada de la creciente inmigración internacional, puede alterar el papel de la educación en la formación de la pareja en España, tal y como se ha observado en países donde la heterogeneidad étnica es un rasgo estructural de la población. En este sentido, es de esperar que el nivel de homogamia e hipergamia femenina de las uniones de los extranjeros varíe en función de la composición por origen de estas parejas. Nuestra hipótesis apunta a una diferenciación, tanto en las pautas de homogamia como en la asimetría por sexo entre los extranjeros de distintas procedencias. Los europeos comunitarios seguirán las mismas tendencias que los españoles, incluso pueden acentuarlas, mientras que las mayores diferencias se observarán en las uniones formadas por africanos y latinoamericanos.

\section{Datos}

Los datos utilizados en esta investigación proceden de la explotación completa del censo de población de 2001 y han sido obtenidos a través del servidor web del Instituto Nacional de Estadística ${ }^{2}$ (www.ine.es). De manera complementaria, se ha utilizado también la muestra de microdatos del $5 \%$ de hogares del mismo censo, con el fin de poder obtener tablas que combinen el nivel de instrucción y el origen de los cónyuges. La estructura de los datos es extremadamente simple: en primer lugar, trabajamos a partir de la distribución del total de parejas en un momento dado (en nuestro caso, 1 de noviembre de 2001) por nivel de instrucción de la mujer y del hombre y año de nacimiento de la mujer $^{3}$. En segundo lugar, obtenemos la distribución por nivel de instrucción y origen de los cónyuges de la generación más joven (1960-1969).

2. Se ha descartado el Movimiento Natural de la Población, que da cuenta de los matrimonios acontecidos anualmente, porque no informa sobre el nivel de instrucción de los cónyuges.

3. No existen diferencias significativas al analizar los datos tomando como referencia la generación de nacimiento del hombre en lugar de la de la mujer. 
Por razones teóricas y prácticas, hemos considerado todos los tipos de unión, sin distinguirlas por su naturaleza de hecho o de derecho. Analíticamente, nuestro objetivo principal es observar las pautas y las tendencias generales de interacción entre los distintos grupos educativos, por lo que tratar las diferencias en función del tipo de unión u otras variables supondría una desviación de dicho objetivo, aunque es materia susceptible de ser tratada en futuras investigaciones. Además, no se observan diferencias significativas entre trabajar con el total de parejas o sólo con los matrimonios, dado que las uniones de hecho apenas suponen el 3,9\% del total, aunque su peso presenta una gran variabilidad entre las generaciones consideradas (de 1,7\% para las generaciones de 1920-1929 a 7,2\% para las generaciones de 1960-1969). Por otra parte, el análisis por separado de las uniones consensuales no arroja resultados fiables, conocidas las diferencias importantes de composición y de naturaleza que se observan entre las uniones de las generaciones más antiguas y las más jóvenes. Por ejemplo, del total de parejas cohabitantes pertenecientes a las generaciones de 1920-1929, sólo en un 8,8\% de los casos ambos miembros eran solteros. Sin embargo, para las generaciones de 1960-1969, esta cifra alcanza el $45,5 \%$ del total. La diversidad de situaciones ocultas tras las uniones consensuales obligaría a tratar por separado cada una de ellas para evitar errores de interpretación.

El nivel de instrucción tomado como referencia es el declarado en la fecha del censo y, por tanto, no se corresponde con el que tenían los cónyuges en el momento de casarse o unirse. Sin embargo, no consideramos que esta decisión, obligada por la disponibilidad de los datos, afecte en demasía a los resultados finales, puesto que el nivel de instrucción adquirido apenas varía después de la celebración del matrimonio, el cual se produce, de manera mayoritaria, al concluir la etapa formativa.

El censo nos proporciona una variable sobre el nivel de instrucción basada en diez categorías, que hemos recodificado en cinco grupos: en primer lugar, agrupamos a todos aquellos que no tienen estudios, sean o no analfabetos; en segundo lugar, a los que disponen de estudios primarios (con un mínimo de cinco años de escolarización); en tercer y cuarto lugares, distinguimos para los estudios secundarios entre bachillerato elemental y superior respectivamente (distinción que, según el calendario académico vigente para las generaciones más jóvenes, se corresponde con la frontera de la escolarización obligatoria), y en quinto y último lugar, agrupamos a todos aquellos con estudios superiores, es decir, a todos los universitarios, con independencia de la duración y del nivel adquirido, diplomatura, licenciatura o doctorado. Teniendo en cuenta la dificultad de diseñar una clasificación igualmente representativa para cada una de las generaciones analizadas, la propuesta finalmente adoptada no sólo resulta operativa desde un punto de vista analítico, sino que también es coherente con las principales divisorias del sistema educativo español. Como veremos a continuación, los resultados validan esta clasificación. Además, con el objetivo de reducir las posibles distorsiones introducidas por esta clasificación, hemos utilizado, en alguno de nuestros modelos, definiciones extendidas de homo- 
gamia, que van más allá de la interacción específica entre dos grupos exactamente iguales.

Tomando el año de nacimiento como referencia, hemos construido cinco grupos decenales de generaciones, de 1920-1929 a 1960-1969, con lo cual hemos cubierto un intervalo de edades en el momento del censo de 32 a 81 años. Aunque no se trate propiamente de un análisis longitudinal, el análisis de las pautas y tendencias en la homogamia educativa basado en datos censales y, por tanto, tomando como referencia distintas edades, no parece excesivamente arriesgado, dado que la educación alcanzada no suele variar después de los 32 años — la edad más temprana considerada —, la divorcialidad no es muy elevada y la viudedad no parece tener una distribución muy desigual en función del grado de instrucción. Es decir, que las parejas formadas por las generaciones consideradas, aun observadas a cierta distancia temporal de cuando fueron formadas, seguirían siendo representativas de su distribución original, como mínimo para mostrar la pauta general de interacción entre los distintos grupos educativos.

El censo informa tanto de la nacionalidad como del país de nacimiento de los individuos y de sus cónyuges. La decisión de identificar a los individuos según su origen a partir de una u otra de estas dos variables resulta una decisión delicada. Por una parte, la nacionalidad se puede modificar, de manera que, considerando sólo a los extranjeros, podemos dejar de lado a los inmigrantes nacionalizados e incluir en cambio a los nacidos en España de ascendencia extranjera. Por otra parte, al considerar exclusivamente el lugar de nacimiento, consideramos tanto a los españoles nacidos en el extranjero como a los inmigrantes extranjeros. Ninguna de las opciones nos ofrece una solución perfecta para identificar con rigor a los inmigrantes. Ante esta disyuntiva, optamos por crear cinco grupos de orígenes utilizando las dos variables simultáneamente. En primer lugar, tenemos a los españoles nacidos en España y, en segundo lugar, a los extranjeros nacidos en el extranjero, que se agrupan en cuatro categorías regionales: los países europeos comunitarios (puesto que el censo es de 2001, consideramos sólo los quince países que formaban parte de la Unión Europea antes de la ampliación al este de 2004), países europeos no comunitarios $^{4}$, una selección de países africanos ${ }^{5}$ y una selección de países latinoamericanos $^{6}$ (aquéllos más representativos de los flujos de inmigración reciente).

\section{Modelos loglineales}

Los modelos loglineales son comúnmente utilizados para analizar las pautas de interacción entre dos o más variables (Knoke y Burke, 1980). A diferencia de los modelos de regresión logística o los modelos de riesgo, desarrollados en el marco del event history analysis, los modelos loglineales poseen algunas carac-

4. República Checa, Eslovaquia, Hungría, Eslovenia, Rumanía, Bulgaria, Polonia, Estonia, Letonia, Lituania, Rusia, Bielorrusia, Yugoslavia, Ucrania y Croacia.

5. Gambia, Senegal, Marruecos, Nigeria y Argelia.

6. Colombia, Ecuador, Bolivia, Perú, República Dominicana y Brasil. 
terísticas especialmente apropiadas para el análisis de la composición de las parejas. En primer lugar, estos modelos no distinguen entre variable dependiente y variables independientes, sino que miden la asociación entre dos o más variables, más allá de los que se relacionarían por la simple intervención del azar, lo cual permite formulaciones teóricas más flexibles. En segundo lugar, los modelos loglineales tratan el mercado matrimonial de forma holística, es decir, considerando todas las interacciones posibles sin necesidad de fragmentarlas para ser adaptadas a otro tipo de técnicas. Se trata, pues, de una visión más cercana a los mercados matrimoniales, lo cual no obliga a fracturar el análisis en múltiples combinaciones o transiciones, que, en la mayoría de casos, son interdependientes entre sí.

En tercer lugar, y no menos importante, los modelos loglineales descomponen jerárquicamente cada uno de los efectos, por ejemplo: el efecto de pertenecer a un grupo A, el efecto de pertenecer a un grupo B y el efecto de pertenecer a A y $\mathrm{B}$ simultáneamente. Es precisamente este último efecto el que puede ser utilizado como un indicador neto de la interacción entre las variables, libre del efecto de la estructura o distribución de los marginales. A partir de aquí, podemos formular todo tipo de hipótesis en torno a las pautas de interacción entre las distintas variables y plantear modelos específicos que respondan a las preguntas que nos interesan, partiendo, por razones de exposición, del modelo de independencia, cuyas frecuencias esperadas se pueden estimar según el modelo siguiente:

$$
\log f_{i q}=\mu_{0}+\mu_{i}+\mu_{i}
$$

Donde $\log f_{i j}$ es el logaritmo natural de la frecuencia esperada de la fila $i$ columna $j ; \mu_{0}$, la constante; $\mu_{i}$, el parámetro para la fila $i, \mathrm{y} \mu_{j}$, el parámetro para la columna $j$.

En términos de homogamia educativa, este modelo asume que no existe relación entre la educación del hombre y la educación de la mujer. En el otro extremo, tenemos el modelo saturado, que asume una interacción específica para cada una de las combinaciones, calculando, por tanto, un parámetro para cada una de ellas. La expresión matemática de este modelo es:

$$
\log f_{i j}=\mu_{0}+\mu_{i}+\mu_{j}+\mu_{i j}
$$

Donde $\mu_{j}$ es el parámetro de la interacción entre la fila $i$ y la columna $j$.

El modelo saturado, aunque tiene la particularidad de reproducir exactamente los datos, no tiene interés analítico, puesto que consume tantos parámetros como interacciones quiere explicar. Se trata, por tanto, de un modelo con nula parsimonia ${ }^{7}$. Sin embargo, entre el modelo independiente y el modelo saturado, existen numerosas combinaciones que, con más o menos parsimonia, tienen interés explicativo en términos substantivos, porque examinan la validez de hipótesis específicas sobre las pautas de interacción entre las variables analizadas.

7. Se denomina parsimonia al grado de sencillez de un modelo: cuanto menos parámetros son necesarios para explicar un fenómeno, más parsimonioso es el modelo. 
En una tabla de contingencia de doble entrada en la que ambas variables comparten exactamente las mismas categorías, es habitual encontrar que las celdas de la diagonal concentren más casos de los que concentrarían por azar. Cuando esto ocurre, es apropiado explorar la condición de casi independencia, que asume independencia en todas las celdas excepto en las de la diagonal. La casi independencia más esquinas $(\mathrm{E})$ es una extensión de esta estructura, que asume que las esquinas, en nuestro caso las celdas $[1,2]$, $[2,1][4,5]$ y $[5,4]$, tampoco satisfacen la condición de independencia y, por tanto, también deben diferenciarse del resto.

Finalmente, la simetría $(S)$ comprueba, para aquellas interacciones entre categorías distintas, si el orden de las variables influye. Si existiera simetría, por ejemplo, las celdas $[4,5]$ y $[5,4]$ mostrarían el mismo nivel de interacción. Para la homogamia educativa, esto significa que la probabilidad de una unión entre personas con distinto nivel de instrucción es independiente del hecho que sea el hombre o la mujer el que tenga mayor o menor nivel académico. Para comprobar, sin embargo, si existe una tendencia uniforme en uno de los sexos para unirse hacia arriba o hacia abajo, calculamos, siguiendo el ejemplo de Mare (1991), el parámetro de asimetría (A).

\section{Resultados}

La tabla 1 muestra, en primer lugar, la estructura educativa por sexo y generación de los cónyuges del total de 8.201.278 parejas analizadas. Las proporciones por nivel académico dan cuenta de la importante expansión educativa experimentada por las generaciones consideradas. Durante el siglo XX, la estructura por nivel de instrucción de la población española se ha expandido como consecuencia de la progresiva universalización del acceso al sistema educativo y de la ampliación del período formativo. El resultado de estos dos procesos ha sido un aumento del nivel educativo medio de los españoles y especialmente de las españolas, que consiguen, en las generaciones más jóvenes, eliminar la tradicional diferencia de género en materia docente. Casi la mitad de los hombres y las mujeres que vivían en pareja en el 2001 y habían nacido entre 1920 y 1929 no tenían estudios, exactamente el 43,18\% de los hombres y el $47,15 \%$ de las mujeres. En cambio, para las generaciones de 1960-1969 la proporción de sin estudios no alcanza el 5\%, ni entre los hombres ni entre las mujeres. Por otro lado, la proporción de hombres y de mujeres con estudios secundarios aumenta espectacularmente conforme las generaciones son más jóvenes. La proporción de hombres con estudios secundarios, combinando las dos categorías de este nivel de instrucción, crece del $15,6 \%$ al $60,5 \%$, y la de las mujeres, del $12,6 \%$ al $59,9 \%$, lo que supone un aumento del $75 \%$ y del $79,6 \%$, respectivamente. Lo mismo ocurre con el nivel de estudios superiores, cuya proporción aumenta del 4,4\% al 17,3\% para los hombres y del 1,8\% al 19,4\% para las mujeres. Así, por primera vez, la proporción de mujeres con estudios superiores de las generaciones de 1960-1969 supera a la de sus cónyuges respectivos. Debemos recordar que, a lo largo de este artículo, no estamos obser- 
Tabla 1. Estructura educativa de los cónyugues y composición educativa de las uniones según generación (España 1920-1969)

\begin{tabular}{lccccc}
\hline Estructura educativa masculina \% & $1920-1929$ & $1930-1939$ & $1940-1949$ & $1950-1959$ & $1960-1969$ \\
\hline Menos de primaria & 43,2 & 36,4 & 20,5 & 9,2 & 4,1 \\
Primaria & 36,7 & 36,6 & 32,3 & 25,0 & 18,0 \\
Secundaria I & 10,2 & 14,0 & 24,3 & 30,7 & 33,0 \\
Secundaria II & 5,4 & 7,1 & 12,1 & 19,5 & 27,5 \\
Estudios superiores & 4,4 & 5,9 & 10,7 & 15,7 & 17,4 \\
\hline Total & 100,0 & 100,0 & 100,0 & 100,0 & 100,0 \\
\hline Estructura educativa femenina \% & $1920-1929$ & $1930-1939$ & $1940-1949$ & $1950-1959$ & $1960-1969$ \\
\hline Menos de primaria & 47,1 & 40,3 & 22,9 & 9,7 & 3,9 \\
Primaria & 38,4 & 39,3 & 37,3 & 27,5 & 16,3 \\
Secundaria I & 9,6 & 13,7 & 26,3 & 34,5 & 34,0 \\
Secundaria II & 3,1 & 4,1 & 7,3 & 15,1 & 26,5 \\
Estudios superiores & 1,8 & 2,7 & 6,1 & 13,1 & 19,4 \\
\hline Total & 100,0 & 100,0 & 100,0 & 100,0 & 100,0 \\
\hline Composición por nivel de insrucción* & $1920-1929$ & $1930-1939$ & $1940-1949$ & $1950-1959$ & $1960-1969$ \\
\hline \% hombre $=$ mujer & 78,6 & 74,6 & 64,6 & 57,6 & 53,4 \\
\% hombre $>$ mujer & 16,0 & 18,8 & 25,3 & 25,7 & 21,5 \\
\% hombre < mujer & 5,4 & 6,6 & 10,1 & 16,6 & 25,1 \\
\hline Total & 100,0 & 100,0 & 100,0 & 100,0 & 100,0 \\
\hline
\end{tabular}

Fuente: censo de población del 2001 (INE).

Nota: la composición por nivel de instrucción se ha hecho considerando a la generación de la mujer.

vando el total de la población, sino sólo aquéllos y aquéllas que convivían en pareja en el año 2001.

En segundo lugar, la tabla 1 informa de la composición de las uniones por nivel educativo y generación. La proporción de uniones homógamas, es decir, aquéllas en las que los cónyuges tienen el mismo nivel de estudios, ha pasado del 78,5\% al 53,3\% entre el primer y el último grupo de generaciones. Se observa así que, paralelamente al incremento del nivel de instrucción de la población, la proporción de parejas homógamas se ha reducido significativamente. Es interesante observar como se ha repartido el incremento de las uniones heterógamas y si se ha alterado la pauta de predominio de la hipergamia femenina (unión en la que la mujer tiene un nivel educativo inferior al del hombre). Ha aumentado el peso de ambas combinaciones de generación en generación, que ha pasado de un $16 \%$ a un $21,5 \%$ las parejas con el hombre más instruido, y de un $5 \%$ a un $25 \%$ las parejas con la mujer más instruida. Sin embargo, como puede apreciarse, el incremento ha sido más importante en el segundo caso, de tal manera que la combinación que era claramente minoritaria para las generaciones más antiguas, compuesta por la mujer más instruida que el hombre, representa, para los nacidos en los años sesenta, una cuarta parte de las uniones, de manera que supera incluso a la combinación contraria. 
Para abordar el análisis combinado de la composición de las parejas por nivel educativo y origen de la generación más joven, 1960-1969, necesitamos conocer la estructura académica de los extranjeros y la composición también académica de sus uniones. En primer lugar, la tabla 2 muestra que los europeos comunitarios son los extranjeros que presentan la estructura más elevada, más incluso que la de los propios españoles. Por su parte, los europeos no comunitarios y los latinoamericanos presentan estructuras educativas parecidas entre sí, que se distinguen de la española fundamentalmente por el peso inferior de los universitarios. Finalmente, en el caso de los africanos, los grupos mayoritarios son los que no tienen estudios primarios y los que sólo tienen estudios primarios. En lo que se refiere a las diferencias por sexo, es de destacar que sólo las españolas superan a los españoles en el porcentaje con estudios superiores. En todos los demás grupos, la estructura educativa de los hombres es ligeramente superior que la de las mujeres del mismo origen (especialmente en el acceso a los estudios universitarios).

En segundo lugar, la tabla 2 muestra la variación de la composición por nivel de instrucción de las parejas en función del sexo y de la composición por nacionalidad de la unión. La proporción de uniones con el mismo nivel de instrucción es mayoritaria para todos los grupos nacionales. Se observan pequeñas diferencias entre ellos, la homogamia educativa es ligeramente superior entre los europeos, tanto comunitarios como no comunitarios. Las diferencias más importantes se observan en la distribución de las parejas heterógamas. Si bien en el caso de españoles y europeos comunitarios el peso de las uniones donde la mujer es más instruida que el hombre es superior a la combinación inversa (como resultado de la pérdida de vigencia del modelo tradicional de hipergamia educativa femenina), en el resto de grupos predominan las uniones donde la mujer es menos instruida que el hombre.

Por otra parte, cabe señalar que la homogamia educativa es mucho más elevada en las uniones entre dos extranjeros de la misma nacionalidad que en las uniones mixtas. Por ejemplo, en el caso de los hombres europeos (del grupo de países comunitarios anteriores a la ampliación), la proporción de homogamia educativa es del $73 \%$ cuando están unidos de forma endógama y sólo del $53 \%$ cuando lo están de forma exógama. Es decir, la heterogamia educativa aumenta cuando la pareja también es heterógama por origen. Aunque nuestro interés no radica en las pautas de intermarriage, sí resulta interesante aclarar que los grupos estudiados están unidos mayoritariamente de manera endógama (es decir, en parejas con dos cónyuges de la misma nacionalidad) con proporciones que superan el $75 \%$, tanto para europeos no comunitarios como para africanos y latinoamericanos. Sólo los europeos comunitarios se desmarcan de esta pauta, puesto que sólo un tercio de los hombres y de las mujeres están unidos con un cónyuge de su misma nacionalidad. Finalmente, es preciso señalar que hemos presentado aquí las proporciones globales de homogamia, pero que éstas son susceptibles de variar entre los distintos niveles educativos, y son presumiblemente los grupos extremos los que mantienen proporciones más elevadas, como veremos a continuación en el apartado de resultados. 
Tabla 2. Estructura educativa de los cónyuges según sexo y grupo de origen, 2001 (España, generación 1960-69)

\begin{tabular}{|c|c|c|c|c|c|}
\hline $\begin{array}{l}\text { Estructura educativa } \\
\text { masculina } \%\end{array}$ & Españoles & $\begin{array}{c}\text { Europeos } \\
\text { occidentales }\end{array}$ & $\begin{array}{l}\text { Europeos } \\
\text { orientales }\end{array}$ & Africanos & Latinoamericanos \\
\hline Menos de primaria & 2,8 & 3,3 & 5,8 & 37,6 & 5,8 \\
\hline Primaria & 36,7 & 36,6 & 32,3 & 25,0 & 18,0 \\
\hline Secundaria I & 10,2 & 14,0 & 24,3 & 30,7 & 33,0 \\
\hline Secundaria II & 5,4 & 7,1 & 12,1 & 19,5 & 27,5 \\
\hline Estudios superiores & 4,4 & 5,9 & 10,7 & 15,7 & 17,4 \\
\hline Total & 100,0 & 100,0 & 100,0 & 100,0 & 100,0 \\
\hline $\begin{array}{l}\text { Estructura educativa } \\
\text { femenina } \%\end{array}$ & Españoles & $\begin{array}{c}\text { Europeos } \\
\text { occidentales }\end{array}$ & $\begin{array}{l}\text { Europeos } \\
\text { orientales }\end{array}$ & Africanos & Latinoamericanos \\
\hline Menos de primaria & 47,1 & 40,3 & 22,9 & 9,7 & 3,9 \\
\hline Primaria & 38,4 & 39,3 & 37,3 & 27,5 & 16,3 \\
\hline Secundaria I & 9,6 & 13,7 & 26,3 & 34,5 & 34,0 \\
\hline Secundaria II & 3,1 & 4,1 & 7,3 & 15,1 & 26,5 \\
\hline Estudios superiores & 1,8 & 2,7 & 6,1 & 13,1 & 19,4 \\
\hline Total & 100,0 & 100,0 & 100,0 & 100,0 & 100,0 \\
\hline $\begin{array}{l}\text { Composición por nivel } \\
\text { de instrucción* }\end{array}$ & Españoles & $\begin{array}{c}\text { Europeos } \\
\text { occidentales }\end{array}$ & $\begin{array}{l}\text { Europeos } \\
\text { orientales }\end{array}$ & Africanos & Latinoamericanos \\
\hline$\%$ hombre $=$ mujer & 78,6 & 74,6 & 64,6 & 57,6 & 53,4 \\
\hline$\%$ hombre $>$ mujer & 16,0 & 18,8 & 25,3 & 25,7 & 21,5 \\
\hline$\%$ hombre $<$ mujer & 5,4 & 6,6 & 10,1 & 16,6 & 25,1 \\
\hline Total & 100,0 & 100,0 & 100,0 & 100,0 & 100,0 \\
\hline
\end{tabular}

Fuente: censo de población del 2001 (INE).

Nota: la composición por nivel de instrucción se ha hecho considerando el origen del hombre.

A continuación, con la aplicación de los modelos loglineales, ahondamos en el análisis de las pautas y tendencias en la composición educativa de las parejas españolas, aislando las restricciones impuestas por la distribución desigual de efectivos por nivel de instrucción. Con todo ello, obtenemos medidas más precisas de la propensión a formar pareja con individuos de igual o distinto nivel de instrucción, para el conjunto de la población y también en función del origen.

La tabla 3 presenta la estructura y los resultados de los principales modelos comprobados, de cuyo ajuste informan los estadísticos Likelihood Ratio $\left(\mathrm{G}^{2}\right)$ y Bayesian Indicator Criteria $(\mathrm{BIC})^{8}$. En ambos casos, cuanto menor es el valor

8. $\mathrm{G}^{2}$ es una variable estadística de uso común que funciona razonablemente bien para localizar efectos significativos en tablas de contingencia en las que el número de casos no supera los 1.500 (Knoke y Burke, 1980). G ${ }^{2}$ es proporcional al número de casos, razón por la cual, cuando aumenta el tamaño muestral, este indicador da prioridad a los modelos más complejos, lo cual complica su interpretación sin normalmente aportar información relevante. BIC solventa parcialmente este problema. Para su cálculo, utiliza el indicador $\mathrm{G}^{2}$ y los grados de libertad. Se trata de un indicador que mide la eficiencia del modelo, al tener en cuenta su capacidad explicativa y los recursos utilizados. 
Tabla 3. G2 y BIC para los modelos seleccionados

\begin{tabular}{lccc}
\hline Modelo & Grados de libertad & Total G2 & Total BIC \\
\hline 1. HC, MC & 80 & $86.743,4$ & 66.401
\end{tabular}

\section{Homogamia}

2. HC, MC, HM

$\begin{array}{lll}64 & 924,2 & 217\end{array}$

3. HC, MC, HM, DbC

$\begin{array}{lll}55 & 208,9 & -419\end{array}$

4. HC, MC, HM, EbC

51

82,1

Simetría

5. HC, MC, $S$

6. HC, MC, S, P

7. HC, MC, S, PC

8. HC, MC, SC, PC

25

577,2

$-247$

Nota: C. Generación (4); H. Nivel de instrucción de los hombres (4); M. Nivel de instrucción de las mujeres (4); D. Parámetros de homogamia (5); E. Parámetros de homogamia más esquinas (9); S. Parámetros de simetría (10); P. Parámetro de asimetría (1); b. Lineal en C.

de estos indicadores, mejor es el ajuste y, por tanto, mejor es la capacidad explicativa del modelo.

Existen diversas alternativas para presentar y comentar los resultados obtenidos mediante la aplicación de modelos loglineales. En este trabajo, por coherencia argumental, hemos decidido clasificar estos modelos en dos grandes bloques. Con el primer bloque, formado por los modelos 2 a 4, damos respuesta a la siguiente pregunta: ¿en qué medida las personas se unen de forma homógama, es decir, con consortes de su mismo nivel de instrucción? El segundo bloque está formado por los modelos 5 a 8, los cuales ponen el acento en la obtención de parámetros para evaluar la vigencia de la hipergamia femenina (pareja en la que la mujer tiene un nivel de instrucción inferior al del hombre) entre las generaciones observadas.

Ninguno de los modelos de ambos bloques tendría razón de ser si, previamente, no comprobáramos, con el modelo 1, que la condición de independencia no se satisface, es decir, que el nivel de instrucción de las personas es relevante en la composición de las parejas y, por tanto, las frecuencias observadas no son simplemente el resultado de la combinación azarosa entre los individuos. Bajo el supuesto de independencia, la probabilidad de una mujer analfabeta de unirse con un hombre con estudios universitarios sería exactamente igual a la de una mujer con estudios universitarios. Pero el deficiente ajuste del modelo $1(\mathrm{BIC}=66401)$ nos obliga a rechazar dicho supuesto e invita a explorar nuevas formulaciones que expliquen mejor los datos observados. Se justifica así la razón de ser de los modelos 2 a 8, cuyos resultados presentamos a continuación para analizar la evolución de los niveles de homogamia educativa y la vigencia del modelo de hipergamia educativa femenina. 


\section{Continuidad y cambio de los niveles de homogamia educativa}

El primer bloque de modelos, del segundo al cuarto, se interesa básicamente por la composición de las parejas en relación con el nivel de instrucción de los cónyuges. El modelo 2 comprueba la siguiente hipótesis: la existencia de una interacción más allá del azar entre el nivel de instrucción de los hombres y el de las mujeres, la cual se mantiene constante entre generaciones. Este modelo mejora el ajuste respecto al modelo de independencia, lo que pone de manifiesto la existencia de una interacción entre los niveles educativos de ambos cónyuges. No obstante, no se trata de un ajuste satisfactorio según los estándares estadísticos, motivo por el cual aplicamos dos modelos más que nos permiten valorar el sentido de dicha interacción.

Los modelos 3 y 4 informan de la intensidad de las uniones homógamas, asumen que existen diferencias generacionales, es decir, que las generaciones más jóvenes no tratan la educación de la misma forma que las más antiguas y mejoran notablemente el ajuste ${ }^{9}$. En términos substantivos, los modelos 3 y 4 nos dicen que, en efecto, existe interacción entre el nivel de instrucción de los hombres y el de las mujeres, especialmente en las celdas de la diagonal (D), modelo 3, o de la diagonal más esquinas (E), modelo 4, que agrupan a las parejas homógamas según utilicemos respectivamente una definición estricta o extendida de homogamia educativa. Además, estos modelos indican que existen diferencias generacionales. En resumen, estos modelos indican que existe, más allá del azar, una pauta específica de interacción entre cónyuges en función de su nivel de instrucción y que en ningún caso esta pauta puede asumirse constante entre las generaciones, es decir, se ha dado un cambio generacional.

A continuación, detallamos estas pautas específicas de interacción conyugal a partir de los parámetros obtenidos con el modelo 4, el que ofrece mejor ajuste. El modelo 4 produce un conjunto de parámetros que evalúan la fortaleza de la diagonal y las esquinas para atraer observaciones o uniones adicionales más allá del azar. Los valores positivos indican cuantas uniones más hay en cada celda respecto a las que hubiera habido bajo el supuesto de independencia. Como se puede comprobar en el gráfico 1, no hay ningún parámetro negativo para ningún nivel y generación, lo que demuestra de entrada que, controladas las desigualdades en la estructura por nivel de instrucción de hombres y mujeres, existe una propensión clara a formar pareja dentro del mismo grupo, aunque dicha propensión varíe en función del grupo y de la generación considerada. La interacción entre los que no tienen estudios es superior a

9. El indicador $\mathrm{BIC}$ alcanza valores negativos $\left(\mathrm{BIC}_{3}=-419 ; \mathrm{BIC}_{4}=-496\right)$, lo que quiere decir que estos modelos son preferibles incluso al modelo saturado. En ambos casos, hemos asumido que la variación entre generaciones evoluciona según una tendencia constante de tipo lineal, lo que nos permite ganar grados de libertad, y, consecuentemente, mejorar la eficiencia del modelo. Asumir esta variación constante de tipo lineal ofrece incluso mejores resultados que estimar un parámetro distinto para cada generación, razón por la cual no hemos considerado oportuna su obtención. 


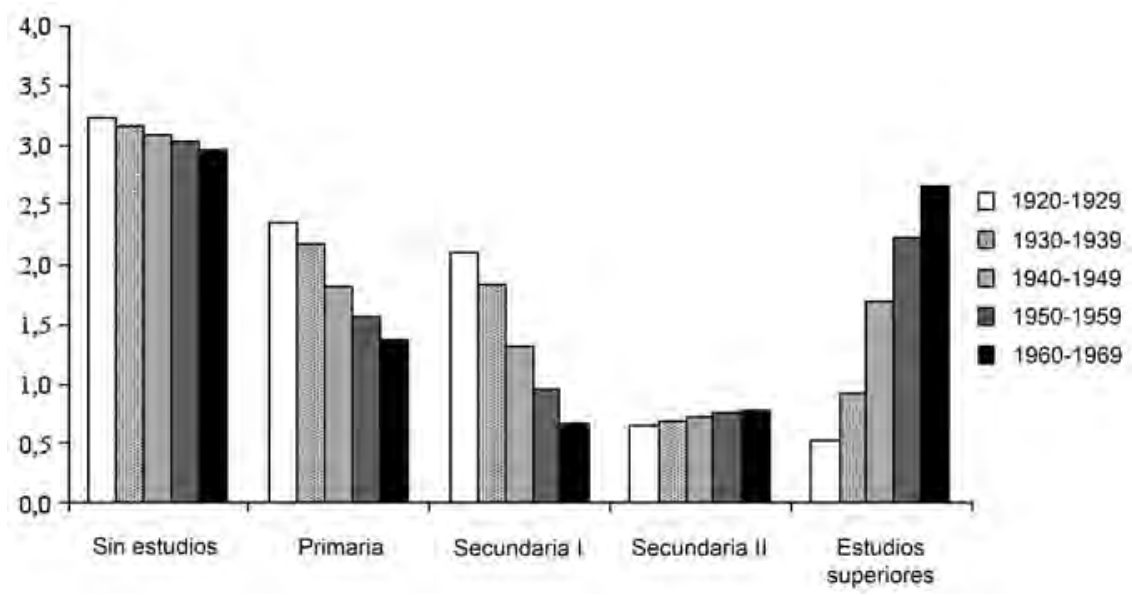

Gráfico 1. Parámetros de homogamia por nivel de instrucción y generación, modelo 4.

cualquier otra y se mantiene más o menos constante por generación. Normalmente, la homogamia educativa en las categorías extremas es más alta, por la simple razón que tienen limitadas sus opciones matrimoniales en un único sentido, ascendente o descendente. Sin embargo, éste no es el caso de la interacción entre los hombres y las mujeres con estudios superiores para las tres primeras generaciones: la interacción entre los más educados aumenta de forma importante de la primera generación a la última. El grado de homogamia entre hombres y mujeres con estudios secundarios más allá de la escolarización obligatoria (secundaria II) presenta unos índices relativamente bajos, que apenas varían entre generaciones. Este hecho obligaría a reconsiderar en futuros trabajos la pertinencia de incluir esta categoría educativa en nuestra clasificación. Y, finalmente, la homogamia para los niveles educativos de primaria y secundaria I disminuyen al unísono entre las generaciones, aunque el grado de interacción es distinto.

\section{Vigencia de la pauta de hipergamia educativa femenina}

Habiendo constatado la tendencia mayoritaria a formar pareja en modo homogámico, en la segunda etapa de este análisis nos interrogamos sobre las parejas que se constituyen entre hombres y mujeres de distinto nivel de instrucción. Nuestro interés principal respecto a estas parejas se concentra en analizar si las pautas de heterogamia masculinas y femeninas son equivalentes. Para las generaciones de la primera mitad del siglo XX, la proporción de mujeres que han formado pareja hipergámicamente, es decir, con un cónyuge más instruido que ellas, es superior a la de los hombres que han escogido a una mujer con nivel de instrucción superior (ver tabla 1). Debemos preguntarnos, sin embar- 


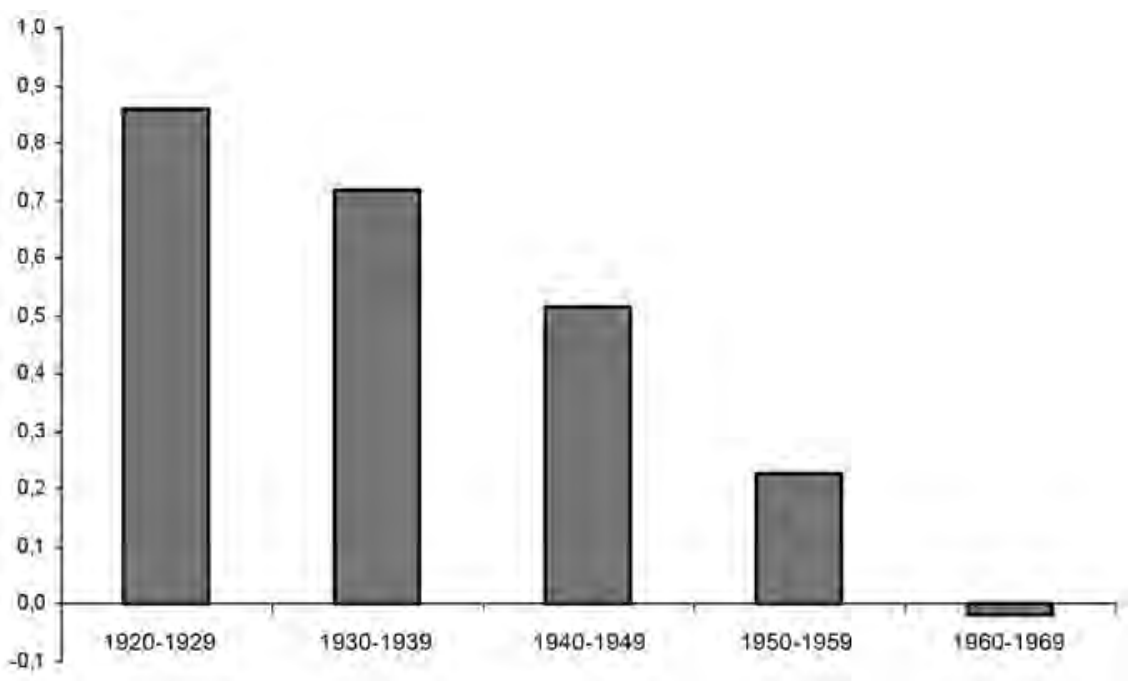

Gráfico 2. Parámetros de asimetría de sexo en la heterogamia por generación, modelo 8.

go, en qué medida esta pauta de hipergamia educativa femenina es producto de las diferencias educativas entre hombres y mujeres. Controlando el efecto de la estructura educativa con la aplicación de los modelos 5 a 8 , conseguiremos explorar el grado real de simetría por sexo.

El modelo 5 asume que las pautas de interacción entre cónyuges son simétricas, que la probabilidad que se produzca una unión entre dos personas con distinto nivel educativo no está afectada por el hecho de que sea el hombre o la mujer el que tenga mayor o menor nivel. El modelo 6 añade un parámetro adicional a esta condición: se trata de un parámetro de asimetría que permite una tendencia uniforme de las mujeres a unirse hacia arriba o hacia abajo (hipergamia o hipogamia, respectivamente) para todas las combinaciones de niveles educativos (Mare, 1991: 22). Tanto el modelo 5 como el 6 mantienen estos parámetros invariables entre generaciones, razón por la cual su ajuste no es el más satisfactorio. El modelo 7 mantiene la simetría constante entre generaciones, pero libera el parámetro de asimetría de esta restricción, lo cual permite su libre movilidad entre generaciones. El indicador BIC es altamente sensible a esta ligera modificación. Con sólo cuatro grados de libertad menos, el BIC transita de 175 para el modelo 8 a -183 para el modelo 7 , prueba suficiente para demostrar que los parámetros añadidos están captando importantes transformaciones entre generaciones. El modelo 8 permite también a los parámetros de simetría variar entre las generaciones, modificación que tiene también repercusión en el BIC, aunque no tan destacada como la observada entre el modelo 6 y 7 . Por lo tanto, concluimos que la introducción de una hipótesis que supone que las pautas masculinas y femeninas de heterogamia 
varían decisivamente en función de la generación de los cónyuges es totalmente pertinente. En efecto, se han producido cambios significativos en el modo en el que hombres y mujeres se aparejan con un cónyuge de nivel educativo inferior o superior.

El parámetro de asimetría obtenido del modelo 8 nos proporciona una medida sintética pero muy ilustrativa sobre la dirección en la que se han producido estos cambios. Libre del efecto de la distribución marginal de la tabla, este parámetro indica que, aún siendo más probables para todas las generaciones, excepto la de 1960-1969, las uniones en las que la mujer tenía un nivel de instrucción menor al de su pareja, esta pauta ha perdido vigencia veloz y constantemente entre las generaciones analizadas, de manera que incluso se ha llegado a invertir el sentido tradicional de la misma en las generaciones más jóvenes (ver gráfico 2).

\section{La composición educativa de las uniones de los extranjeros}

El considerable aumento de los flujos de inmigración extranjera que ha experimentado España desde mediados de los años noventa, introduce la conveniencia de abordar el estudio de la homogamia educativa teniendo en cuenta también el origen de los cónyuges. Aquí se analiza el papel que desempeña el nivel de instrucción en la formación de la pareja de los extranjeros en España para la generación más joven, la que comprende a las parejas formadas por dos cónyuges nacidos entre 1960 y 1969.

En primer lugar, vemos como varían los niveles de homogamia según el origen combinado de los cónyuges. Para ello, utilizamos un modelo loglinear (complementario del modelo 4 anteriormente presentado, porque simplemente añade a su formulación primigenia el origen de los cónyuges) para calcular un factor que mide la variación en el nivel de homogamia educativa de cada combinación de orígenes en relación con el de las parejas formadas por un español y una española (la categoría de referencia) ${ }^{10}$. El gráfico 3 representa estos factores que toman valores positivos cuando la homogamia es más intensa en esa combinación que en la de referencia, y valores fraccionarios cuando la homogamia es menos intensa. Se observa claramente como los niveles de homogamia de los europeos, tanto occidentales como orientales, tienden a ser superiores a los de las parejas formadas por dos cónyuges españoles, mientras que para africanos y latinoamericanos son claramente inferiores. Además de estas diferencias por origen, el gráfico 3 también permite apreciar las ligeras diferencias que se observan entre las uniones exógamas y endógamas. Es el caso, sobre todo, de los latinoamericanos, que muestran como mientras la combinación entre un español y una latinoamericana tiende a ser tan poco homógama como la de dos latinoamericanos, en cambio la unión entre un latinoa-

10. Este modelo obtiene los siguientes parámetros de ajuste: grados de libertad $=538 ; \mathrm{G}^{2}=$ $2.016,5 ; \mathrm{BIC}=-5.459,1$. 


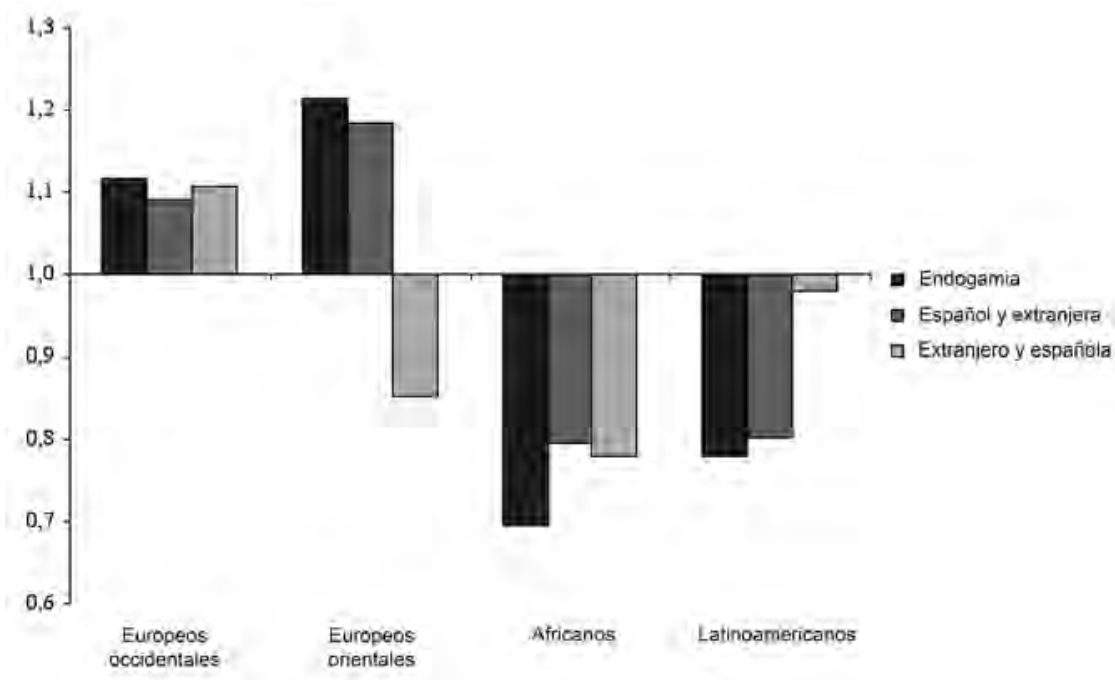

Gráfico 3. Factor de variación de los niveles de homogamia según la composición por nacionalidad de la pareja $($ referencia $=1)$.

mericano y una española resulta ser prácticamente tan homógama como la de los españoles. En este sentido, los hombres latinoamericanos, cuando se unen con una española, no presentan pautas diferenciadas en relación con las de los españoles. El caso de los europeos orientales parece funcionar en el sentido contrario al de los latinoamericanos. Sin embargo, este factor debe ser tomado con cautela, por el limitado número de uniones de esa combinación.

En segundo lugar, una vez constatadas las diferencias de homogamia, nos interesa conocer qué pauta de heterogamia por sexo siguen estos extranjeros. También aquí utilizamos un modelo loglinear (equivalente al modelo 8 anteriormente presentado, pero incluyendo esta vez el origen de los cónyuges además de su nivel educativo) que calcula los parámetros de hipergamia femenina para cada combinación de orígenes ${ }^{11}$. El gráfico 4 muestra los parámetros de asimetría obtenidos, que son positivos cuando las uniones heterógamas tienden más hacia la hipergamia femenina, es decir, tienden a ser formadas por la combinación «hombre más instruido que la mujer», y que son negativos en el caso que predomine la hipogamia femenina. Los resultados indican que, para la generación 1960-1969, la pauta de hipergamia femenina sigue vigente en las uniones formadas por africanos y latinoamericanos. Sin embargo, los europeos presentan una clara superación de este modelo tradicional y el predominio de las uniones formadas por una mujer más instruida que el hombre es

11. Este modelo obtiene los siguientes parámetros de ajuste: grados de libertad $=536 ; \mathrm{G}^{2}=$ $1.546,9 ; \mathrm{BIC}=-5.486,8$. 


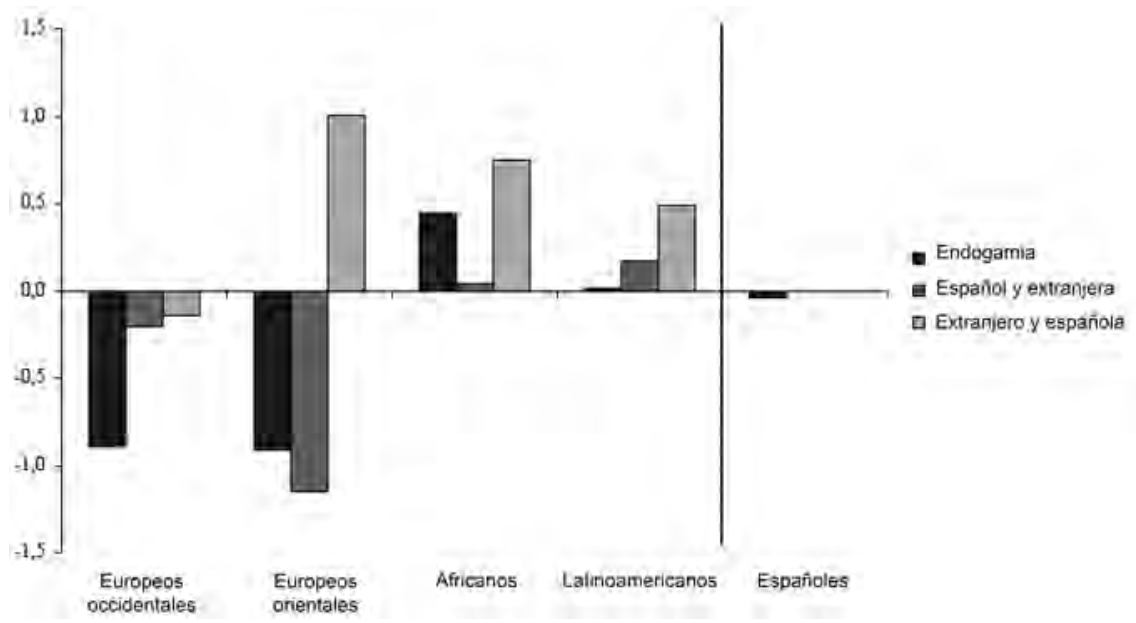

Gráfico 4. Parámetros de asimetría de sexo en la heterogamia según composición por nacionalidad de la pareja.

más contundente incluso que en las uniones formadas por dos españoles (que, como hemos visto, superaban por primera vez, en la generación de 1960-1969, a la combinación contraria).

\section{Conclusiones}

Al empezar este artículo, acudíamos al concepto de aleatoriedad para explorar hasta qué punto las pautas y tendencias en homogamia educativa en la España contemporánea eran simplemente el resultado de una correspondencia azarosa entre los cónyuges, sólo condicionada por la distribución por nivel de instrucción de hombres y mujeres. Esta idea, que se mantiene en el ámbito estricto de lo hipotético, es la que nos ha servido de hilo argumental en esta investigación, en la que hemos mostrado que, más allá de los determinantes estructurales impuestos por la distribución por sexos y generaciones del nivel de instrucción, la educación de los cónyuges es un aspecto relevante en la composición de las parejas.

En primer lugar, constatamos que, para todas las generaciones analizadas, 1920-1969, existe una propensión mayoritaria a formar parejas homógamas. La pareja formada por un hombre y una mujer de nivel educativo equivalente es el modelo estándar en España. Dicho modelo ha perdido fuerza durante el siglo $\mathrm{XX}$, en un proceso de cambio generacional remarcable, aunque sigue siendo la pauta dominante.

En segundo lugar, desvelamos, mediante el análisis específico por niveles educativos, como esta tendencia general a la reducción de los niveles de homogamia esconde diferencias significativas entre grupos educativos. Los niveles de 
homogamia entre los universitarios aumentan significativamente de generación en generación, mientras se reducen en los grupos de educación inferior.

En tercer lugar, no podemos dejar de considerar los cambios acontecidos en las parejas heterógamas, entre las que el predominio de la hipergamia femenina ha perdido definitivamente vigencia. Teniendo en cuenta la reducción de las diferencias educativas por sexos, las uniones en las que la mujer tiene un nivel de instrucción inferior al del hombre son cada vez menos frecuentes, lo que revela un cambio en la pauta tradicional de formación de la pareja.

En cuarto y último lugar, el análisis de la interacción entre la composición por nivel de instrucción y por origen de las parejas revela que la condición de extranjero altera significativamente las pautas de composición educativa de las uniones, en relación con las de los españoles. En el caso de las parejas formadas por los europeos, tanto comunitarios como no comunitarios, se refuerzan los niveles de homogamia y se supera claramente el patrón clásico de hipergamia femenina. En cambio, en el caso de los africanos y los latinoamericanos (más las mujeres que los hombres), sus uniones tienden a ser más heterógamas y, concretamente, más hipérgamas desde el punto de vista de las mujeres, es decir, más tradicionales en lo que se refiere a las diferencias de género.

Estos resultados han sido obtenidos mediante el análisis específico de la correlación de los niveles de instrucción de los cónyuges. Sin embargo, su interpretación podría completarse y enriquecerse ampliando el análisis a otras características que son susceptibles de interactuar con la educación. Podemos pensar, por ejemplo, en considerar simultáneamente el nivel de instrucción de los cónyuges y el de sus progenitores; podemos contrastar los indicadores de educación con indicadores de nivel socioeconómico y podemos también evaluar la influencia del calendario nupcial sobre las pautas de homogamia, preguntándonos, por ejemplo, si el proceso de retraso de las edades al matrimonio puede estar acentuando la propensión a formar pareja entre los más formados. El análisis combinado de estos elementos contribuiría a realizar una descripción completa de la composición de las parejas españolas, necesaria para el desarrollo posterior del estudio de las implicaciones sociales de las pautas matrimoniales en la estructura social en España.

\section{Referencias bibliográficas}

BECKER, G. (1987). Tratado sobre la familia. Madrid: Alianza.

BirKelUND, G.E. y HeLDAL, J. (2003). «Who marries whom? Educational homogamy in Norway». Demographic Research (Max Planck Institute), 8(1).

BLACKWELL, D.L. (1998). «Marital homogamy in the United States: The influence of individual and parental education». Social Science Research, 27, 159-188.

Blossfeld, H.-P. y Timm, A. (eds.) (2003). Who marries whom? Educational systems as marriage markets in modern societies. Vol. 12. Holanda: Kluwer Academic Publishers.

CABré, A. (1993). «Volverán tórtolos y cigüeñas». En: GarRido, L. y Gil CalVo, E. (eds.). Estrategias familiares. Madrid: Alianza, 113-131.

Carabaña, J. (1994). «La constante homogamia educativa». Economia y Sociedad, 11, 43-66. 
CORTINA, C. (2004). Nivell educatiu, origen geogràfic i nacionalitat en la composició de les parelles a Espanya: Una aproximació a l'estudi de l'homogàmia i l'endogàmia. Barcelona: Universitat Autònoma de Barcelona. Departament de Geografia. Memoria de investigación.

Cortina , C., Esteve, A. y Domingo, A., (2008). «Marriage Patterns of the ForeignBorn Population in a New Country of Immigration: The Case of Spain». International Migration Review, 42 (4), 877-902.

Cortina, C.; Esteve, A. y Domingo, A. (2006). «Crecimiento y singularidades demográficas de los matrimonios de extranjeros en España». Papers de Demografia, 307 Migraciones, 20, 75-105.

Esteve, A. (2005). «Tendencias en homogamia educacional en México: 1970-2000». Estudios Demográficos y Urbanos, 59-20(2), 341-362.

Esteve, A. y McCAA, R. (2007). "Homogamia educativa en México y Brasil, 19702000: Pautas y tendencias». Latin American Research Review, 42(2), 56-85.

Esteve , A.; CortinA, C. (2006). "Changes in Educational Assortative Mating in Contemporary Spain». Demographic Research, 14(17), 405-428.

Forsé, M. y ChaUvel, L. (1995). «L'évolution de l'homogamie en France». Revue Française de Sociologie, XXXVI, 123-142.

GONZÁLEZ, M.J. (2001). The interplay between ocupational career and family formation in Spain. Tesis de doctorado presentada en el Social and Political Sciences Department, European University Institute (mimeo).

- (2003). "Who marries whom in Spain?». En: Blossfeld, H.-P.; Timm, A. (eds.). Who marries whom? Educational systems as marriage markets in modern societies. Vol. 12. Holland: Kluwer Academic Publishers, p. 57-78.

Gordon, M. (1964). Assimilation in American Life. Oxford University Press.

Halpin, B. y CHAN, T.W. (2003). «Educational homogamy in Ireland and Britain: trends and patterns». British Journal of Sociology, 54(4), 473-495.

Kalmijn, M. (1991). "Status homogamy in the United States». American Journal of Sociology, 97, 496-523.

- (1998). "Intermarriage and homogamy: Causes, patterns, trends». Annual Review of Sociology, 24, 395-421.

KATRNÁK, T. (2005). Educational Assortative Mating in the Czech Republic, Slovakia and Hungary between 1976 and 2003 (mimeo).

KnOKe, D. y Burke, P. (1980). Log Linear Models. Beverly Hills: Sage Publications.

MARE, R.D. (1991). "Five decades of assortative mating». American Sociological Review, 56(1), 15-32.

MCCAA, R. (1993). «Ethnic intermarriage and gender in New York City». Journal of Interdisciplinary History, 24(2), 207-231.

MERTON, R.K. (1941). "Intermarriage and the social structure: Fact and theory». Psyquiatry, 4: 361-374.

PAGNINI, D.L. y Morgan, S.P. (1990). «Intermarriage and social distance among U.S. immigrants at the turn of the century». American Sociological Review, 96, 405-432.

OPPENHEIMER, V.K. (1994). "Women's rising employment and the future of the family in industrial societies». Population and Development Review, 20(2), 293-342.

- (1998). "A Theory of Marriage Timing». American Journal of Sociology, 94(3), 563-591.

PAgnini, D.L. y Morgan, S.P. (1990). «Intermarriage and social distance among U.S. immigrants at the turn of the century». American Sociological Review, 96, 405-432. 
Portes, A. (1993) «The New Second Generation: Segmented Assimilation and Its Variants among Post-1965 Immigrant Youth». Annals of the American Academy of Political Sciences, 53, 75-98.

QIAN, Z. (1998). "Changes in assortative mating. The impact of age and education, 1970, 1990». Demography, 35(3), 279-292.

SCHWARTZ, C. y MARE, R.D. (2005). "Trends in educational assortative marriage from 1940 to 2003». Demography, 42(4), 621-646.

Smits, J.; Ultee, W. y LAMMERS, J. (1998). «Educational homogamy in 65 countries: an explanation of differences in openness using country-level explanatory variables». American Sociological Review, 63, 264-285. 